\title{
Total Thyroidectomy and Low-Risk Thyroid Cancer: Are We Treating the Surgeon or Patient?
}

\author{
Amanda M. Laird, $\mathrm{MD}^{1,2}$, and Toni Beninato, $\mathrm{MD}$, $\mathrm{MS}^{1,2}$ \\ ${ }^{1}$ Division of Surgical Oncology, Department of Surgery, Rutgers Cancer Institute of New Jersey, New Brunswick, NJ; \\ ${ }^{2}$ Rutgers Robert Wood Johnson Medical School, New Brunswick, NJ
}

Surgical treatment of thyroid cancer and the extent of surgery for thyroid cancer is an ongoing debate. One thing that is clear, however, is that thyroid cancer requires carefully planned treatment. An operation for thyroid cancer should completely remove the tumor as well as involved adjacent structures and lymph nodes with the least possible morbidity while reducing risk of recurrence and improving overall survival. What distinguishes thyroid cancer from other malignancies is that there is not a "one size fits all" approach to treatment, and appropriate management requires counseling of the patient such that they can make an informed decision about treatment choice. Unfortunately, there are no large, randomized clinical trials to compare surgical strategies. Current guidelines from the American Thyroid Association (ATA) state that thyroid lobectomy is an option for surgical treatment of follicularderived thyroid cancers when they are less than $4 \mathrm{~cm}$, with no extrathyroidal extension present, and when they are clinically node negative. ${ }^{1}$ This is based on existing evidence that less than total thyroidectomy yields equivalent overall survival (OS) to total thyroidectomy, accepting that the rate of local recurrence may be higher.

The study by Colombo et al. ${ }^{2}$ supports and adds to the existing literature regarding long-term outcomes of follicular-derived thyroid cancer. These data, which are collected over a 38-year period, compare total thyroidectomy (TT) and thyroid lobectomy (TL) for low- and

(C) Society of Surgical Oncology 2021

First Received: 25 March 2021

Accepted: 31 March 2021;

Published Online: 17 April 2021

A. M. Laird, MD

e-mail: amanda.laird@cinj.rutgers.edu intermediate-risk thyroid cancers using the endpoint eventfree survival. Event-free survival was defined as time from surgery to detection of either persistent or recurrent disease. They stratified patients into ATA low-risk and intermediate-risk groups. Low-risk patients were more likely to be event free after TT versus TL (95\% vs $87.5 \%$ ) after a follow-up of 79.7 months. Intermediate-risk patients undergoing TT versus TL were also more likely to be event free (89\% vs $50 \%)$ with a follow-up of 113.1 months. Complication rates, specifically permanent injury to the recurrent laryngeal nerve and permanent hypocalcemia, were higher in patients undergoing TT. Unfortunately, there are no data regarding overall survival (OS) in the current study. The authors conclude that TT should be recommended over TL based on likelihood of recurrence.

These data, in reality, add to what we already know about surgery for thyroid cancer, i.e., that OS is not impacted by extent of surgery in selected patients with lowrisk thyroid cancer despite potential for a higher risk of recurrence. The greater number of events in the intermediate-risk group in the current study is not surprising given that these patients have tumor characteristics which would classify them as ATA intermediate risk. Thus, these would be patients ultimately considered for completion thyroidectomy with or without postoperative radioactive iodine ablation. Data from the National Cancer Database revealed that, in appropriately risk-stratified patients, there was no difference in OS between TT and TL. ${ }^{3}$ Similarly, data from two studies using the Survival, Epidemiology, and End Results (SEER) registry reaffirmed that there is no difference in OS in appropriately selected patients despite surgical approach. ${ }^{4,5}$ A more limited operation avoids the morbidity of a total thyroidectomy and potentially obviates the need for thyroid hormone replacement since a portion of native thyroid gland remains intact. Complication rates 
with respect to hematoma, permanent recurrent laryngeal nerve injury, and permanent hypocalcemia are definitively lower with TL compared with TT. ${ }^{1}$

Because of the excellent outcome in management of the majority of thyroid cancers independent of operation selection, the remaining issues are patient preference, surgeon preference, and the influence of patient comorbidities on choice of operation. Though not yet well studied in thyroid cancer, shared decision-making is a concept used in the management of other lower-risk malignancies. The strategy has been employed in thyroid cancer using a conversation aid to guide patient decision-making between active surveillance versus surgery. ${ }^{6}$ A survey of thyroid cancer patients receiving radioactive iodine (RAI) revealed lower satisfaction when they felt they did not have a choice. ${ }^{7}$ Decisions for extent of surgery are also influenced by the treating physician's own feelings toward the disease. In a survey-based study by McDow et al., 345 physicians responded regarding their beliefs in treatment of thyroid cancer. Overall, the group preferred TT if they were undergoing surgery, and they believed TT would be preferred by the patient if recommended by the surgeon or endocrinologist, for "fear of cancer," and for "peace of mind." ${ }^{8}$ Finally, patient condition may influence either decision to operate or extent of operation. Frailty as a measure of fitness for operation is associated with increased medical and surgical complications following inpatient thyroidectomy. ${ }^{9}$

In his 2011 address as President of the American Association of Endocrine Surgeons, Dr. Douglas Evans highlighted some of the key issues in the management of papillary thyroid cancer (PTC). ${ }^{10}$ Once more sensitive indicators of recurrence of PTC, i.e., ultrasound and measurements of thyroglobulin ( $\mathrm{Tg})$, became available, there was greater interest in performing larger operations to eradicate clinically evident disease. He also drew comparisons to medullary thyroid cancer (MTC) as well as highlighted differences in management. While we are comfortable tolerating persistently mildly elevated calcitonin levels after surgery, the tendency is to actively pursue mild persistent $\mathrm{Tg}$ elevations by repeat ultrasounds, $\mathrm{Tg}$ monitoring, and, perhaps, biopsy or surgery. In 2011, recommendations for thyroid cancer management were different, and the majority of cancers were treated with total thyroidectomy with or without central compartment lymphadenectomy. Now, 10 years later, we know that, for small or otherwise low-risk cancers, an operation of this magnitude does not impact survival. So, the primary question that remains is: Are we treating the patient, or are we treating our beliefs as surgeons?
Thyroid lobectomy is a valid treatment for low-risk thyroid cancer. We accept the need for future potential reoperation in these patients because recurrence does not influence overall survival. Perhaps it is time to set aside the need for surgeon "peace of mind" in exchange for appropriate patient counseling. We have an opportunity to reiterate the need for long-term follow-up and for patients to have a greater role in the decision-making regarding the operation selected.

DISCLOSURE The authors declare no conflicts of interest.

\section{REFERENCES}

1. Haugen BR, Alexander EK, Bible KC, et al. 2015 American Thyroid Association management guidelines for adult patients with thyroid nodules and differentiated thyroid cancer: the American Thyroid Association Guidelines Task Force on Thyroid Nodules and Differentiated Thyroid Cancer. Thyroid. 2016;26(1):1-133.

2. Colombo C, De Leo S, Di Stefano M, et al. Total thyroidectomy versus lobectomy for thyroid cancer: single center data and literature review. Ann Surg Oncol. 2021. https://doi.org/10.1245/ s10434-020-09481-8.

3. Adam MA, Pura J, Gu L, et al. Extent of surgery for papillary thyroid cancer is not associated with survival: an analysis of 61,775 patients. Ann Surg. 2014;260(4):601-5 (discussion 605-607).

4. Mendelsohn AH, Elashoff DA, Abemayor E, St John MA. Surgery for papillary thyroid carcinoma: is lobectomy enough? Arch Otolaryngol Head Neck Surg. 2010;136(11):1055-61.

5. Haigh PI, Urbach DR, Rotstein LE. Extent of thyroidectomy is not a major determinant of survival in low- or high-risk papillary thyroid cancer. Ann Surg Oncol. 2005;12(1):81-9.

6. Brito JP, Moon JH, Zeuren R, et al. Thyroid cancer treatment choice: a pilot study of a tool to facilitate conversations with patients with papillary microcarcinomas considering treatment options. Thyroid. 2018;28(10):1325-31.

7. Wallner LP, Reyes-Gastelum D, Hamilton AS, Ward KC, Hawley ST, Haymart MR. Patient-perceived lack of choice in receipt of radioactive iodine for treatment of differentiated thyroid cancer. J Clin Oncol. 2019;37(24):2152-61.

8. McDow AD, Roman BR, Saucke MC, et al. Factors associated with physicians' recommendations for managing low-risk papillary thyroid cancer. Am J Surg. 2020. https://doi.org/10.1016/j.a mjsurg.2020.11.021.

9. Xu D, Fei M, Lai Y, Shen Y, Zhou J. Impact of frailty on inpatient outcomes in thyroid cancer surgery: 10-year results from the U.S. national inpatient sample. J Otolaryngol Head Neck Surg. 2020;49(1):51.

10. Evans DB. Papillary carcinoma of the thyroid: balancing principles of oncology with emerging technology. Surgery. 2011;150(6):1015-22.

Publisher's Note Springer Nature remains neutral with regard to jurisdictional claims in published maps and institutional affiliations. 